\section{Brain MRI in patients with diffuse psychiatric/neuropsychological syndromes in systemic lupus erythematosus}

Yoshiyuki Arinuma, ${ }^{1}$ Hirotoshi Kikuchi, ${ }^{2}$ Tatsuhiko Wada, ${ }^{1}$ Tatsuo Nagai, ${ }^{1}$ Sumiaki Tanaka, ${ }^{1}$ Hiroshi Oba, ${ }^{3}$ Shunsei Hirohata ${ }^{1}$

To cite: Arinuma $Y$, Kikuchi $\mathrm{H}$, Wada $\mathrm{T}$, et al. Brain MRI in patients with diffuse psychiatric/ neuropsychological syndromes in systemic lupus erythematosus. Lupus Science \& Medicine 2014;1: e000050. doi:10.1136/lupus2014-000050

Received 17 July 2014 Revised 24 September 2014 Accepted 25 September 2014

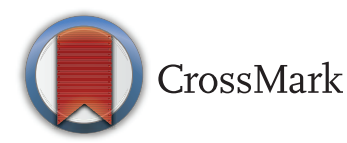

\section{${ }^{1}$ Department of}

Rheumatology and Infectious Diseases, Kitasato University School of Medicine, Kanagawa, Japan

${ }^{2}$ Department of Internal Medicine, Teikyo University School of Medicine, Tokyo, Japan

${ }^{3}$ Department of Radiology, Teikyo University Hospital, Tokyo, Japan

\section{Correspondence to} Dr Yoshiyuki Arinuma; y-arinuma@med.kitasati-u.ac. jp

\begin{abstract}
Background: Manifestations in neuropsychiatric systemic lupus erythematosus (NPSLE), especially active diffuse NPSLE syndromes, are some of the most difficult complications of the disease. For the evaluation and the diagnosis of central nervous system manifestations, including NPSLE, MRI is a very useful tool to detect the various abnormalities. However, the relationship between brain MRI findings and clinical variables has not yet been clarified in patients with diffuse NPSLE.
\end{abstract}

Objectives: The aim of this study is to investigate the pathogenesis of diffuse NPSLE, by comparing various parameters such as serum autoantibodies and cytokines in cerebrospinal fluid (CSF) with abnormal findings revealed on brain MRIs in patients with diffuse NPSLE.

Methods: Fifty-three patients with diffuse NPSLE admitted to our University Hospital from 1992 to 2012 were exhaustively enrolled in this study. Their medical charts and brain MRI scans were reviewed. The relationship of MRI abnormalities with various parameters was analysed.

Results: As many as 25 of 53 patients $(47.2 \%)$ had abnormal MRI findings. MRI findings improved after treatment in 10 of 17 patients for whom follow-up studies were available. MRI abnormalities were not correlated with age at the onset of diffuse NPSLE. However, the disease duration of SLE was significantly longer in patients with abnormal MRI findings $(p=0.0009)$. MRI abnormalities were not significantly associated with serum autoantibodies. However, there were significant elevations of the CSF protein level $(p=0.0106)$ and the CSF interleukin 6 level $(p=0.0225)$ in patients with abnormal MRI findings. Patients with MRI abnormalities showed significantly higher overall mortality ( $p=0.0348)$.

Conclusions: The results revealed that MRI abnormalities in diffuse NPSLE might be heterogeneous with regard to their reversibility. These data also indicate that patients with diffuse NPSLE and MRI abnormalities have more severe inflammation in the central nervous system related to the activity of diffuse NPSLE, as evidenced by poorer prognosis.

\section{KEY MESSAGES}

- The brain MRI abnormalities in patients with diffuse NPSLE are heterogeneous.

- Diffuse NPSLE patients with brain MRI abnormalities have poorer prognosis due to severe SLE activities.

- The severity of diffuse NPSLE could be associated with the vasculitis in brain according to the increased CSF IL-6 and protein level in patients with brain MRI abnormalities.

\section{INTRODUCTION}

Systemic lupus erythematosus (SLE) is a chronic inflammatory disease characterised by the expression of a variety of autoantibodies, involving multiple organs. ${ }^{1}$ Neuropsychiatric manifestations in SLE (NPSLE), which occur in $37-95 \%$ of patients, are some of the most difficult complications of the disease. ${ }^{2-8}$

NPSLE includes the neurological syndromes of the central, peripheral and autonomic nervous system and the psychiatric syndromes. However, diagnoses and research in NPSLE were cumbersome because there was no standardised nosology. In 1999, the American College of Rheumatology (ACR) established nomenclature and case definitions of NPSLE as nosology for clinical descriptions and research in SLE. ${ }^{9}$ This nomenclature provides 19 neuropsychiatric syndromes in SLE, including 12 syndromes of central nervous system (CNS) disease and 7 of peripheral nervous system disease. In CNS diseases, brain MRI is one of the most useful imaging techniques in identification of CNS lesions developing from various disorders, including NPSLE. ${ }^{10}$

Recently, two studies have documented the brain MRI findings in large numbers of patients with only active NPSLE classified according to the 1999 ACR nomenclature and case definitions for NPSLE syndromes. ${ }^{11}{ }^{12}$ 
They found that brain MRI abnormalities were absent in almost half of the patients with active NPSLE, supporting the immune-mediated pathogenesis for such active NPSLE. Nevertheless, they also indicated that several brain MRI findings were obviously identified in active patients with NPSLE. However, although caused by heterogeneous aetiologies, the relationship of the development of brain MRI abnormalities with certain types of NPSLE or autoantibodies has, to our knowledge, not yet been described.

According to the 1999 ACR nomenclature and case definitions, diffuse psychiatric/neuropsychological syndromes in NPSLE (including anxiety disorder, acute confusional state, cognitive dysfunction, mood disorder, psychosis) are diffuse CNS diseases unlike neurological syndromes originated from focal CNS lesions (including cerebrovascular disease, demyelinating syndrome, headache, aseptic meningitis, chorea, seizures and myelopathy). These diffuse NPSLE syndromes are associated with the presence of autoantibodies against neuronal cells in serum as well as cerebrospinal fluid (CSF). ${ }^{13} 14$ Interleukin (IL)-6 is one of the proinflammatory cytokines of which measurement in CSF is useful to diagnose diffuse NPSLE whenever infection and stroke are discriminated. ${ }^{15}$ Nevertheless, to our knowledge, no previous reports have delineated the association between brain MRI abnormalities and certain types of NPSLE or severity of the disease, especially in diffuse psychiatric/ neuropsychological syndromes in active NPSLE (diffuse NPSLE).

The aim of this study was to clarify the pathogenesis of diffuse NPSLE. The characteristics of brain MRI findings in patients with diffuse NPSLE were compared to investigate the relationship of brain MRI findings with various clinical factors, such as serum autoantibodies, CSF cytokines, cell count, and glucose and protein levels in patients with diffuse NPSLE, especially focusing on inflammation in the CNS.

\section{MATERIALS AND METHODS \\ Patients}

A total of 53 patients, who had been admitted to Kitasato University Hospital and Teikyo University Hospital due to diffuse NPSLE as the first event and who had received brain MRI scan examinations from 1992 to 2012, were exhaustively enrolled in this retrospective study. All the patients fulfilled the 1982 ACR revised classification criteria for SLE. ${ }^{16}$ and also presented at least one of the five manifestations of diffuse NPSLE in the 1999 ACR nomenclature and case definitions for NPSLE syndromes. ${ }^{9}$ Patients diagnosed with infectious diseases, metabolic diseases or toxicoses were excluded from the present study. All 53 patients were treated with high-dose corticosteroids with or without steroid pulse therapy. Based on the efficacy of immunosuppressive therapy, their neuropsychiatric manifestations were attributed to SLE. When the disease activity could not be controlled only by corticosteroids, then immunosuppressive agents such as cyclophosphamide and/or rituximab were used. Antipsychotics were minimally used during the acute phases of the diseases. All the patients' clinical data including the prognoses were reviewed based on their medical records until 1 September 2012. The disease activity of SLE was evaluated according to the British Isles Lupus Assessment Group-2004 index, ${ }^{17}$ which can be converted into a numerical score. ${ }^{18}$ A flare-up of SLE was defined as the elevation of disease activities when grades A or B of the British Isles Lupus Assessment Group-2004 index were satisfied, that in turn required an increase in the corticosteroid dose with or without the addition of an immunosuppressive drug. The date of flare-up of SLE or death was determined from the medical records. This study has been approved by the ethics committees in Kitasato University School of Medicine and in Teikyo University School of Medicine.

\section{Autoantibodies and IL-6 measurement}

Anti-dsDNA antibodies were evaluated by radioimmunoassay or ELISA, anti-Sm, anti-cardiolipin (CL), and anti-CL $\beta 2$-glycoprotein I complex antibodies were detected by ELISA. Lupus anticoagulant was determined by dilute Russell's viper venom time. These antibodies were measured by the manufacturers who each defined their own cut-off values themselves. Antiphospholipid (anti-PL) antibodies included anti-CL antibodies, anti-CL $\beta 2$-glycoprotein I complex antibodies and lupus anticoagulant; and the positivity of anti-PL antibodies was defined as the presence of one or more of three tests as positive. Antiribosomal $\mathrm{P}$ protein (anti-ribo $\mathrm{P}$ ) antibodies were determined by ELISA as previously described. ${ }^{19}$ CSF IL-6 is an effective measure in diagnosing diffuse NPSLE at the cut-off value of $4.3 \mathrm{pg} / \mathrm{mL} .^{15}$ CSF IL-6 testing was done by ELISA or bioassay using IL-6 dependent cell line MH60.BSF2. ${ }^{20}$

\section{MRI acquisition}

All MRI scans were performed using a $1.0 \mathrm{~T}$ or $1.5 \mathrm{~T}$ MR scanner along with the usual procedures in clinical practice within a few days of the occurrence of diffuse NPSLE. MRI included T1-weighted and T2-weighted images, fluid-attenuated inversion recovery (FLAIR) images and diffusion-weighted images. In some patients, gadolinium enhancement was performed on T1-weighted or FLAIR images. Whenever possible, a follow-up examination of the brain MRI was done 2-4 weeks after treatment. For the analysis of the reversibility, the results from the first follow-up were used. All the MRI scans were evaluated by expert neuroradiologists.

\section{Statistical analysis}

Comparison between patients with MRI abnormalities and those without significant MRI findings was performed using the Mann-Whitney U test, Fisher's exact test and logistic regression analysis where appropriate. Effects of MRI abnormalities on relapse of SLE or as related to mortality were analysed by the log-rank test 
with the Kaplan-Meier method, using JMP V.5.1.2. (SAS Institute, Cary, North Carolina, USA).

\section{RESULTS}

Relationship of brain MRI abnormalities with neuropsychiatric manifestations

Of the 53 patients, brain MRI revealed abnormalities in the parenchyma or the meninges in 25 patients $(47.2 \%)$. As shown in table 1 , there were no significant differences in gender, ages at the onset of diffuse NPSLE, or the disease activity between patients with MRI abnormalities and those without MRI abnormalities, although ages at the onset of diffuse NPSLE appeared to be higher in patients with MRI abnormalities $(p=0.0625)$. Consistently, the disease duration of SLE was significantly longer in patients with MRI abnormalities (76.4 \pm 111.4 months, mean \pm SD) than in those without MRI abnormalities (19.8 \pm 55.2 months) $(\mathrm{p}=0.0009)$. Moreover, patients with MRI abnormalities contained higher numbers of events of relapsed SLE than did those without MRI abnormalities $(p=0.0525)$ (table 1). There were no significant differences in the distribution of various neuropsychiatric manifestations, including acute confusional state, psychosis, anxiety disorder, mood disorder and cognitive dysfunction between the two groups (table 1). There were 11 patients with diffuse NPSLE who also had seizures. However, the complication of seizures was not significantly correlated with abnormal high-density lesions on brain MRI.

The most prevalent abnormal MRI findings in patients with diffuse NPSLE included multiple small white matter hyperintensities (WMHs) on T2-weighted or
FLAIR images (22 patients, $88 \%$ ). Eighteen of $22 \mathrm{WMHs}$ were of patients with 1 to 10 multiple scattered lesions of less than $10 \mathrm{~mm}$ in diameter located in the subcortical area. Two patients had approximately $30 \mathrm{~mm}$ of diffuse infiltrative lesions in the white matter. While grey matter hyperintensities were detected in the hippocampus, basal ganglia, cerebellum and brain stem in seven patients (28\%), most patients (six of seven, $86 \%$ ) also exhibited complications of WMH (table 2). The lesions of the hippocampus showed diffuse hyperintensities. The lesions on the basal ganglia showed diffuse hyperintensities in two patients and punctate intensities in one patient. Cerebellar lesions consisted of multiple spotted changes. These results indicated that WMHs were the most prevalent abnormalities on brain MRI scans in this study.

Seventeen of the 23 patients with MRI abnormalities were also followed up after treatment. MRI abnormalities at the onset of diffuse NPSLE improved after the successful treatment in 10 of those 17 patients $(55.8 \%)$, but did not change in six patients or slightly exacerbated in one patient, despite the amelioration of diffuse NPSLE. The most prevalent changes in improved lesions were the reduction of the involved size. A few lesions had disappeared as revealed in the follow-up examinations. These data suggest that brain MRI abnormalities in diffuse NPSLE might consist of at least two different pathogeneses.

\section{Relationship of brain MRI abnormalities with various parameters in serum and CSF}

Autoantibodies play an important role in pathogenesis in NPSLE. ${ }^{14} 2122$ However, serum anti-DNA, anti-Sm,

Table 1 Characteristics of patients at the onset of diffuse NPSLE

\begin{tabular}{|c|c|c|c|c|}
\hline \multirow[b]{2}{*}{ Characteristics } & \multirow[b]{2}{*}{ Patients $(\mathrm{N}=53)$} & \multicolumn{2}{|c|}{ Brain MRI abnormal findings } & \multirow[b]{2}{*}{ p Value } \\
\hline & & Absent $(n=28)$ & Present $(n=25)$ & \\
\hline Gender (male/female) & $8 / 45$ & $3 / 25$ & $5 / 20$ & $0.4527^{*}$ \\
\hline Age (years) (mean $\pm S D)$ & $38.9 \pm 17.1$ & $35.1 \pm 17.2$ & $43.1 \pm 16.3$ & $0.0625 \dagger$ \\
\hline Disease duration (months) (mean $\pm S D$ ) & $47.1 \pm 90.3$ & $19.8 \pm 55.2$ & $76.4 \pm 111.4$ & $0.0009 \dagger$ \\
\hline Follow-up period (months) (mean $\pm S D$ ) & $72.6 \pm 44.6$ & $81.2 \pm 74.5$ & $62.8 \pm 64.7$ & $0.3312 \dagger$ \\
\hline Initial presentation of SLE & $33(62.3 \%)$ & $21(75.0 \%)$ & $12(48.0 \%)$ & $0.0525 \dagger$ \\
\hline Mean of total BILAG-2004 index score & 14.2 & 14.6 & 13.9 & $0.6148 \dagger$ \\
\hline \multicolumn{5}{|l|}{ Neuropsychiatric manifestations } \\
\hline ACS & 37 & 17 & 20 & $0.1474^{*}$ \\
\hline Psychosis & 6 & 5 & 1 & $0.1959^{*}$ \\
\hline$A D$ & 3 & 3 & 0 & $0.2380^{*}$ \\
\hline MD & 12 & 7 & 5 & $0.7499^{*}$ \\
\hline CD & 6 & 2 & 4 & $0.4042^{*}$ \\
\hline Complication of seizures & 11 & 4 & 7 & $0.3126^{\star}$ \\
\hline Hypertension & 3 & 1 & 2 & $1.0000^{*}$ \\
\hline Hyperlipidaemia & 0 & 0 & 0 & \\
\hline Diabetes mellitus & 3 & 2 & 1 & $1.0000^{*}$ \\
\hline
\end{tabular}

*Fisher's exact test.

†Mann-Whitney U test.

ACS, acute confusional state; AD, anxiety disorder; BILAG, British Isles Lupus Assessment Group; CD, cognitive dysfunction; MD, mood disorder; NPSLE, neuropsychiatric systemic lupus erythematosus; SLE, systemic lupus erythematosus. 
Table 2 Characteristics of MRI abnormalities and their relevance with neuropsychiatric presentations

\begin{tabular}{|c|c|c|c|c|c|c|c|}
\hline Brain MRI findings & Patients ( $\mathrm{N}=53$ ) & ACS & Psychosis & AD & MD & CD & Seizure \\
\hline Abnormal MRI ${ }^{*}$ & 25 & 20 & 1 & 0 & 5 & 4 & 7 \\
\hline WMH & 22 & 18 & 1 & 0 & 4 & 4 & 7 \\
\hline Multiple punctate & 18 & 15 & 1 & 0 & 3 & 0 & 6 \\
\hline Diffuse infiltrative & 2 & 1 & 0 & 0 & 1 & 0 & 1 \\
\hline Haemorrhagic infarction & 2 & 2 & 0 & 0 & 0 & 0 & 0 \\
\hline GMH & 7 & 5 & 0 & 0 & 2 & 1 & 4 \\
\hline Basal ganglia & 2 & 1 & 0 & 0 & 1 & 0 & 2 \\
\hline Hippocampus & 3 & 2 & 0 & 0 & 1 & 1 & 3 \\
\hline Cerebellum & 1 & 1 & 0 & 0 & 0 & 0 & 1 \\
\hline Haemorrhagic infarction & 2 & 2 & 0 & 0 & 0 & 0 & 0 \\
\hline GMH alone & 1 & 1 & 0 & 0 & 0 & 0 & 0 \\
\hline $\mathrm{WMH}+\mathrm{GMH}$ & 6 & 4 & 0 & 0 & 2 & 1 & 4 \\
\hline Meningeal & 2 & 2 & 0 & 0 & 0 & 0 & 0 \\
\hline Atrophic & 14 & 11 & 1 & 1 & 4 & 3 & 3 \\
\hline Abnormal MRI & 9 & 8 & 0 & 0 & 2 & 2 & 3 \\
\hline \multicolumn{8}{|l|}{ Severity } \\
\hline Minimal & 8 & 7 & 1 & 0 & 0 & 1 & 0 \\
\hline Moderate & 5 & 4 & 0 & 0 & 2 & 0 & 2 \\
\hline Marked & 12 & 9 & 0 & 0 & 3 & 3 & 5 \\
\hline
\end{tabular}

*All MRI abnormalities other than cortical atrophy found in all conditions including T1, T2-weighted images, and FLAIR. Minimal including one to three punctate lesions of $\mathrm{WMH}$, moderate including four to six punctate lesions of WMH or meningeal lesions alone, and marked including diffuse infiltrative lesions, haemorrhagic infarction or GMH.

ACS, acute confusional state; $A D$, anxiety disorder; $C D$, cognitive dysfunction; GMH, grey matter hyperintensities; FLAIR, fluid-attenuated inversion recovery; $\mathrm{MD}$, mood disorder; $\mathrm{WMH}$, white matter hyperintensities.

anti-ribo P, or anti-PL antibodies were not significantly associated with the presence of brain MRI abnormalities (figure 1). CSF data were available for 41 patients. In the CSF leucocyte count, there were no differences between patients with MRI abnormalities (39.2 \pm 85.0 cells $\left./ \mathrm{mm}^{3}\right)$ and those without MRI abnormalities (10.9 \pm 18.9 cells $\left./ \mathrm{mm}^{3}\right) \quad(\mathrm{p}=0.3623)$. The CSF glucose level in patients with MRI abnormalities was $60.7 \pm 27.7 \mathrm{mg} / \mathrm{dL}$ and in those without MRI abnormalities was 68.9 $\pm 39.1 \mathrm{mg} / \mathrm{mL}$, respectively, but was not significantly

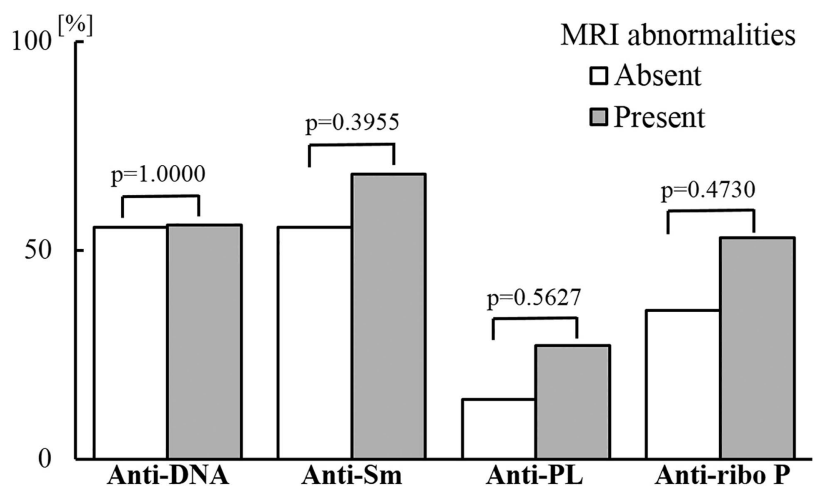

Figure 1 Association of MRI findings and serum autoantibodies in diffuse neuropsychiatric systemic lupus erythematosus. Anti-PL, anti-phospholipid antibody; anti-ribo $P$, anti-ribosomal $P$ protein antibody. Anti-PL included anticardiolipin IgG antibody, anti-cardiolipin $\beta 2$ glycoprotein I antibody and/or lupus anticoagulant. Statistical significance was evaluated by Mann-Whitney U test. different $(\mathrm{p}=0.5888)$. Whereas the CSF protein level was significantly elevated in patients with MRI abnormalities $(199.2 \pm 44.1 \mathrm{mg} / \mathrm{dL})$, it was significantly lower in those without MRI abnormalities $(37.5 \pm 8.6 \mathrm{mg} / \mathrm{dL})$ $(p=0.0106)$. CSF IL-6 was significantly higher in patients with brain MRI abnormalities than in those without MRI abnormalities $(\mathrm{p}=0.0225)$ (figure 2$)$. The data suggest that CSF IL-6 might be involved in the development of brain high intensity lesions detected on MRI in diffuse NPSLE.

\section{Relevance of brain MRI abnormalities with the prognosis of} diffuse NPSLE

Finally, we retrospectively examined the influences of MRI abnormalities on the prognosis of patients with

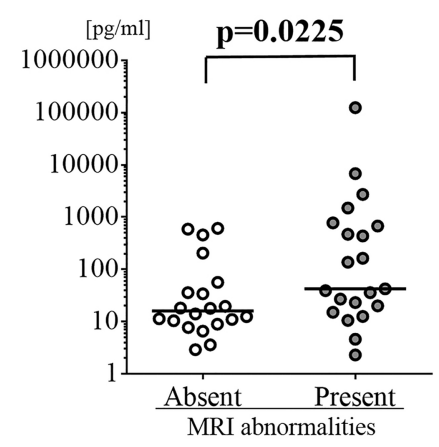

Figure 2 Relationship between cerebrospinal fluid IL-6 and abnormal MRI findings in diffuse neuropsychiatric systemic lupus erythematosus. Statistical significance was evaluated by Mann-Whitney $\mathrm{U}$ test. 
diffuse NPSLE. During the observation periods (72.6 \pm 44.6 months, mean \pm SD), 12 of the 53 patients $(22.6 \%)$ experienced exacerbation of general SLE activities requiring an increase in their doses of corticosteroids, with or without the addition of immunosuppressive drugs. Patients without flare-ups of SLE were censored on the date of their last visit. Moreover, two of four patients who died from causes unrelated to SLE were censored on the date of death. As shown in figure 3, the presence of brain MRI abnormalities did not influence the exacerbation of SLE ( $\mathrm{p}=0.8462)$. The 5-year, 10-year and 15-year mortality rates of the 53 patients were $84.5 \%, 84.5 \%$ and $72.4 \%$, respectively (figure $4 \mathrm{~A}$ ). Thus, 9 of the 53 patients $(17.0 \%)$ died during the observation periods. The survival duration of the nine patients from the onset of diffuse NPSLE was 20.2 \pm 50.0 months (mean $\pm \mathrm{SD}$ ). Four of these nine patients, died of causes unrelated to SLE disease activity (three of pneumonia and one of rupture of an aortic aneurysm); and the remaining five patients died of the complications of active SLE, including pulmonary hypertension, catastrophic anti-PL syndrome, thrombotic thrombocytopenic purpura, pneumatosis intestinalis, and cerebritis. Thus, all of the dead patients had severe disease activities or had complications by immunosuppressive therapy such as corticosteroids which had been used due to persistent high disease activities. Brain MRI abnormalities significantly increased the overall mortality rate in patients with diffuse NPSLE (figure 4B).

\section{DISCUSSION}

Previous studies reported that brain MRI abnormalities were observed in $42.9-84.0 \%$ of the patients with NPSLE. $^{11} 12$ 23-26 ${ }^{12}$ However, most studies contained patients with neurological syndromes as well as diffuse NPSLE. ${ }^{11} 12$ 23-26 Focal CNS manifestations included in neurological syndromes usually show various abnormal densities on brain MRI. ${ }^{2}$ For example, cerebrovascular disease, caused by anti-PL antibodies, present abnormal intensity lesions on brain MRI. ${ }^{27}$ Thus, the features on

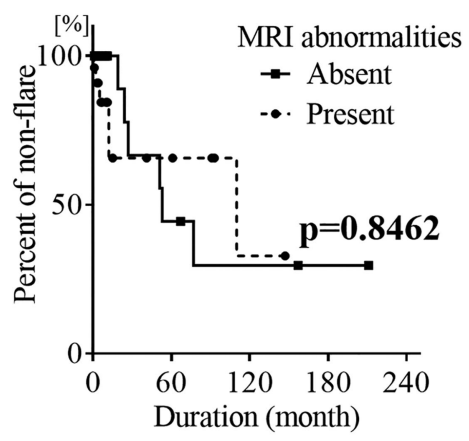

Figure 3 Kaplan-Meier analysis of the effect of MRI findings on the flare of general systemic lupus erythematosus activities in diffuse neuropsychiatric systemic lupus erythematosus. Statistical significance was evaluated by log-rank test.
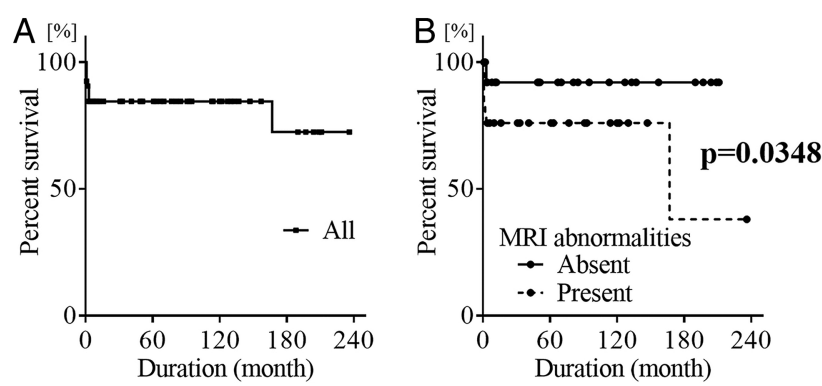

Figure 4 Kaplan-Meier analysis of the effect of MRI findings on the mortality in diffuse neuropsychiatric systemic lupus erythematosus. (A) Overall mortality in all the patients. (B) Effect of MRI findings on overall mortality. Statistical significance was evaluated by log-rank test.

brain MRI in diffuse NPSLE have not been precisely explored. The results in the present study revealed that $47.2 \%$ of patients with diffuse NPSLE had abnormal lesions revealed on brain MRI, even though they did not present with any focal neurological manifestations. In this regard, Castellino et $a l^{28}$ showed that abnormal brain MRIs were observed in $55.8 \%$ of the patients with diffuse NPSLE, which was not significantly different from patients without NPSLE $(36.6 \%)$.

The most frequently observed abnormal lesions in brain MRI were small WMHs in subcortical areas in the present study, consistent with previous studies. ${ }^{11} 12 \quad 2325$ Although Castellino et $a l^{28}$ also demonstrated that WMH was detected in $59.1 \%$ of patients with diffuse NPSLE, such lesions might not be specific for diffuse NPSLE, and have been observed in patients with SLE without NPSLE. ${ }^{28}$ More importantly, MRI abnormalities decreased or disappeared after treatment in some patients, whereas such lesions did not change at all in spite of improvement of diffuse NPSLE in others. The data indicate that brain MRI abnormalities resulted from at least two different mechanisms: one leading to reversible lesions, and the other to irreversible lesions. It would be important to explore the influences of various factors on the development of such reversible or irreversible MRI abnormalities.

A variety of autoantibodies have been shown to be involved in the pathogenesis of diffuse NPSLE. ${ }^{14} 212229$ However, anti-DNA, anti-Sm, anti-PL or anti-ribo $\mathrm{P}$ antibodies were not significantly associated with abnormal brain MRI lesions. Even though anti-PL might cause the irreversible lesions detected on brain MRI, anti-PL was not correlated with the presence of the irreversible brain MRI lesions in the present study. However, CSF IL-6 level was significantly correlated to the presence of brain MRI abnormalities in patients with diffuse NPSLE. Therefore, although CSF IL-6 was above the cut-off level in all the patients, CSF IL-6 was significantly higher in patients with MRI abnormalities. Previous studies disclosed that IL-6 is produced by neurons in diffuse NPSLE. ${ }^{30}$ It is therefore likely that CSF IL-6 might consist of IL-6 derived from neurons as well as that 
derived from abnormal MRI lesions, presumably caused by vasculitis. ${ }^{31}$ The severity classified by the pattern of MRI abnormalities was not associated with certain types of diffuse NPSLE or CSF IL-6 level, indicating that the results of brain MRI abnormalities after being affected by various factors were heterogeneous. However, the presence of brain MRI abnormalities was significantly related to the higher level of CSF IL-6. If the inflammation was caused by vasculitis involving CSF IL-6 elevation, the vasculitis could be classified into two types based on the reversibility of MRI abnormalities, and the reversibility might affect the degree of damage due to vasculitic inflammation. In this regard, anti-N-methyl-D-aspartate receptor subunit NR2 (anti-NR2) antibodies, which have been shown to be elevated in CSF from patients with diffuse NPSLE, ${ }^{14} 1617$ have also been demonstrated to bind endothelial cells and induce production of proinflammatory cytokines, including IL-6. ${ }^{32}$ Therefore, anti-NR2 might cause vasculitis in some patients. Further studies would be important to examine the relationship of abnormal brain MRI lesions with anti-NR2 in serum and CSF of patients with diffuse NPSLE. Moreover, the significant elevation of CSF protein level in patients with MRI abnormalities implies the presence of more severe damage to the blood-brain barrier involving vasculitis. From the results of MRI reversibility, CSF IL-6 and protein level, CNS inflammation in diffuse NPSLE could be divided into three types depending on the presence and the reversibility of brain MRI abnormalities: (1) mild vasculitis with MRI reversibility, (2) moderate or more severe vasculitis without MRI reversibility, and (3) non-vasculitic inflammation without MRI abnormalities.

\section{CONCLUSIONS}

The presence of brain MRI abnormalities significantly increased the mortality, although it did not affect the rate of relapse of general SLE activities. Most of the causes of death were complications of SLE itself or infectious diseases, presumably caused by immunosuppressive therapy for SLE. Therefore, patients with diffuse NPSLE with brain MRI abnormalities had more severe disease and thus affected mortality. It should be noted, however, that patients with brain MRI abnormalities were older and had longer disease duration of SLE. Taken together, brain MRI abnormalities involving vasculitis could correlate to the severity of diffuse NPSLE, which was independent from the overall SLE disease activity.

There are a few limitations to the present study. First, the number of patients with follow-up MRI was small. A larger number of patients should be studied to determine the reversibility of abnormal brain MRI findings. Second, anti-NR2 could be measured in only a limited number of patients. The relationship between anti-NR2 and abnormal brain MRI should be examined on the basis of a larger number of patients. Finally, the present study was unable to incorporate the findings of brain atrophy due to the lack of appropriate age-matched control. Since brain atrophy has been found to be prevalent in patients with SLE, ${ }^{12} 1923$ its relevance with various types of NPSLE warrants further investigation.

Acknowledgements The authors thank Robert E Brandt, Founder, CEO and CME, of MedEd Japan for editing the manuscript.

Contributors All authors contributed to the conception and design of the study, acquisition and/or interpretation of data, drafting the article or revising it critically for important intellectual content and approved the final version of the manuscript.

Competing interests None.

Patient consent Obtained.

Ethics approval Ethics committees in Kitasato University School of Medicine and in Teikyo University School of Medicine.

Provenance and peer review Not commissioned; externally peer reviewed.

Data sharing statement No additional data are available.

Open Access This is an Open Access article distributed in accordance with the Creative Commons Attribution Non Commercial (CC BY-NC 4.0) license, which permits others to distribute, remix, adapt, build upon this work noncommercially, and license their derivative works on different terms, provided the original work is properly cited and the use is non-commercial. See: http:// creativecommons.org/licenses/by-nc/4.0/

\section{REFERENCES}

1. Ruiz-Irastorza G, Khamashta MA, Castellino G, et al. Systemic lupus erythematosus. Lancet 2001;357:1027-32.

2. Gibson T, Myers AR. Nervous system involvement in systemic lupus erythematosus. Ann Rheum Dis 1975;35:398-406.

3. Harris EN, Hughes GR. Cerebral disease in systemic lupus erythematosus. Springer Semin Immunopathol 1985;8:251-66.

4. Ainiala $\mathrm{H}$, Hietaharju $\mathrm{A}$, Loukkola J, et al. Validity of the new American College of Rheumatology criteria for neuropsychiatric lupus syndromes: a population-based evaluation. Arthritis Rheum 2001;45:419-23.

5. Brey RL, Holliday SL, Saklad AR, et al. Neuropsychiatric syndromes in lupus: prevalence using standardized definitions. Neurology 2002;58:1214-20.

6. Hanly JG, McCurdy G, Fougere L, et al. Neuropsychiatric events in systemic lupus erythematosus: attribution and clinical significance. $J$ Rheumatol 2004;31:2156-62.

7. Sanna G, Bertolaccini ML, Cuadrado MJ, et al. Neuropsychiatric manifestations in systemic lupus erythematosus: prevalence and association with antiphospholipid antibodies. J Rheumatol 2003;30:985-92.

8. Sibbitt WL Jr, Brandt JR, Johnson CR, et al. The incidence and prevalence of neuropsychiatric syndromes in pediatric onset systemic lupus erythematosus. J Rheumatol 2002;29:1536-42.

9. ACR Ad Hoc Committee on Neuropsychiatric Lupus Nomenclature. The American College of Rheumatology nomenclature and case definitions for neuropsychiatric lupus syndromes. Arthritis Rheum 1999;42:599-608.

10. Bertsias GK, loannidis JP, Aringer M, et al. EULAR recommendations for the management of systemic lupus erythematosus with neuropsychiatric manifestations: report of a task force of the EULAR standing committee for clinical affairs. Ann Rheum Dis 2010;69:2074-82.

11. Luyendijk J, Steens SC, Ouwendijk WJ, et al. Neuropsychiatric systemic lupus erythematosus: lessons learned from magnetic resonance imaging. Arthritis Rheum 2011;63:722-32.

12. Steup-Beekman GM, Zirkzee EJ, Cohen D, et al. Neuropsychiatric manifestations in patients with systemic lupus erythematosus: epidemiology and radiology pointing to an immune-mediated cause. Ann Rheum Dis 2013;72(Suppl 2):ii76-9.

13. Bluestein HG, Williams GW, Steinberg AD. Cerebrospinal fluid antibodies to neuronal cells: association with neuropsychiatric manifestations of systemic lupus erythematosus. Am J Med 1981;70:240-6.

14. Isshi K, Hirohata S. Differential roles of the anti-ribosomal $P$ antibody and antineuronal antibody in the pathogenesis of central nervous system involvement in systemic lupus erythematosus. Arthritis Rheum 1998;41:1819-27. 
15. Hirohata S, Kanai Y, Mitsuo A, et al. Accuracy of cerebrospinal fluid IL-6 testing for diagnosis of lupus psychosis. A multicenter retrospective study. Clin Rheumatol 2009;28:1319-23.

16. Tan EM, Cohen AS, Fries JF, et al. The 1982 revised criteria for the classification of systemic lupus erythematosus. Arthritis Rheum 1982;25:1271-7.

17. Isenberg DA, Rahman A, Allen E, et al. BILAG 2004. Development and initial validation of an updated version of the British Isles Lupus Assessment Group's disease activity index for patients with systemic lupus erythematosus. Rheumatology (Oxford) 2005;44:902-6.

18. Stoll T, Stucki G, Malik J, et al. Association of the Systemic Lupus International Collaborating Clinics/American College of

Rheumatology Damage Index with measures of disease activity and health status in patients with systemic lupus erythematosus. $J$ Rheumatol 1997;24:309-13.

19. Isshi K, Hirohata S. Association of anti-ribosomal P protein antibodies with neuropsychiatric systemic lupus erythematosus. Arthritis Rheum 1996;39:1483-90.

20. Hirohata S, Miyamoto T. Elevated levels of interleukin-6 in cerebrospina fluid from patients with systemic lupus erythematosus and central nervous system involvement. Arthritis Rheum 1990;33:644-9.

21. DeGiorgio LA, Konstantinov KN, Lee SC, et al. A subset of lupus anti-DNA antibodies cross-reacts with the NR2 glutamate receptor in systemic lupus erythematosus. Nat Med 2001;7:1189-93.

22. Arinuma $Y$, Yanagida T, Hirohata S. Association of cerebrospinal fluid anti-NR2 glutamate receptor antibodies with diffuse neuropsychiatric systemic lupus erythematosus. Arthritis Rheum 2008;58:1130-5.

23. Karassa FB, loannidis JP, Boki KA, et al. Predictors of clinical outcome and radiologic progression in patients with neuropsychiatric manifestations of systemic lupus erythematosus. Am J Med 2000;109:628-34.

24. Sanna G, Piga M, Terryberry JW, et al. Central nervous system involvement in systemic lupus erythematosus: cerebral imaging and serological profile in patients with and without overt neuropsychiatric manifestations. Lupus 2000;9:573-83.

25. Appenzeller S, Vasconcelos Faria A, Li LM, et al. Quantitative magnetic resonance imaging analyses and clinical significance of hyperintense white matter lesions in systemic lupus erythematosus patients. Ann Neurol 2008;64:635-43.

26. McCune WJ, MacGuire A, Aisen A, et al. Identification of brain lesions in neuropsychiatric systemic lupus erythematosus by magnetic resonance scanning. Arthritis Rheum 1988;31:159-66.

27. Galli M, Luciani D, Bertolini G, et al. Lupus anticoagulants are stronger risk factors for thrombosis than anticardiolipin antibodies in the antiphospholipid syndrome: a systematic review of the literature. Blood 2003;101:1827-32.

28. Castellino G, Padovan M, Bortoluzzi A, et al. Single photon emission computed tomography and magnetic resonance imaging evaluation in SLE patients with and without neuropsychiatric involvement. Rheumatology (Oxford) 2008;47:319-23.

29. Yokoyama T, Fujii T, Kondo-Ishikawa S, et al. Association between anti-U1 ribonucleoprotein antibodies and inflammatory mediators in cerebrospinal fluid of patients with neuropsychiatric systemic lupus erythematosus. Lupus 2014;23:635-42.

30. Hirohata S, Hayakawa K. Enhanced interleukin-6 messenger RNA expression by neuronal cells in a patient with neuropsychiatric systemic lupus erythematosus. Arthritis Rheum 1999:42:2729-30

31. Maiolini A, Otten M, Hewicker-Trautwein M, et al. Interleukin-6, vascular endothelial growth factor and transforming growth factor beta 1 in canine steroid responsive meningitis-arteritis. BMC Vet Res 2013;9:23.

32. Yoshio $\mathrm{T}$, Okamoto $\mathrm{H}$, Hirohata $\mathrm{S}$, et al. IgG anti-NR2 glutamate receptor autoantibodies from patients with systemic lupus erythematosus activate endothelial cells. Arthritis Rheum 2013;65:457-63. 